\title{
Molecular Dynamics Study on Structural Relaxation Processes in Amorphous Germanium $^{* 1}$
}

\author{
Shigetaka Imabayashi ${ }^{* 2}$ and Manabu Ishimaru \\ Department of Materials Science and Engineering, Kyushu Institute of Technology, Kitakyushu 804-8550, Japan
}

\begin{abstract}
The structural relaxation of amorphous germanium was examined by molecular dynamics simulations based on the empirical Tersoff interatomic potential. Although the Tersoff potential overestimated both the melting and glass transition temperatures, it was able to reproduce the structural relaxation behavior. The potential energy decreases with thermal annealing below the glass transition temperature, but occasionally increases during structural relaxation. The mean square displacement of atoms also increases in these periods. These changes were attributed to cooperative atomic motion during the structural relaxation. Atomic trajectories revealed that structural changes are induced by spatially- and temporally-inhomogeneous atomic motions: atomically mobile and immobile regions coexist during structural relaxation. [doi:10.2320/matertrans.M2017036]
\end{abstract}

(Received February 6, 2017; Accepted March 10, 2017; Published April 14, 2017)

Keywords: amorphous, germanium structural relaxation, molecular dynamics simulation, empirical interatomic potential

\section{Introduction}

Graphite is currently used as the anode material of lithium-ion batteries, but the development of materials with high electrical capacity continues with the aim of improving battery performance. The theoretical electric capacities of $\mathrm{Si}$ and Ge are $4200 \mathrm{mAh} / \mathrm{g}\left(\mathrm{Li}_{22} \mathrm{Si}_{5}\right)$ and $1624 \mathrm{mAh} / \mathrm{g}\left(\mathrm{Li}_{22} \mathrm{Ge}_{5}\right)$, respectively, much larger than that of graphite $(372 \mathrm{mAh} / \mathrm{g}$ for $\mathrm{LiC}_{6}$ ), and therefore these materials are anticipated as anode materials for the next generation of lithium-ion batteries. However, large volume changes $(>400 \%)$ are induced in $\mathrm{Si}$ and Ge during charge (lithiation) and discharge (de-lithiation), and consequently exfoliation from the collector and pulverization of these materials occur ${ }^{1}$. Nanoporosification is one possible way to solve this problem. Amorphous materials inherently contain free volume, and porous materials can be fabricated by recrystallizing them ${ }^{2-4)}$. In fact, Okugawa et $a l .{ }^{5)}$ confirmed that nanoporous structures can be realized in Ge by electron-beam-induced crystallization. It was also found that the structural relaxation of amorphous $\mathrm{Ge}(a-\mathrm{Ge})$ occurs during aging at room temperature. The electron-beam-induced structures of crystallized Ge depend on the aging time: coarse crystal grains are formed in short-period aged specimens, while a uniform nanoporous structure is formed in long-period aged ones. It has also been confirmed that hexagonal Ge, which is a metastable phase, may be formed by controlling the structural relaxation of amorphous $\mathrm{Ge}^{6)}$. Although structural relaxation plays an important role in controlling nanostructure, the details of the process on the atomic scale are not clear.

Molecular dynamics (MD) simulation is a powerful technique for obtaining static information such as on atomic level structures and defects as well as on dynamic behavior such as atomic vibration in short timescales. MD methods can be roughly divided into (1) the first principles MD method, which does not use experimental parameters, and (2) the clas-

\footnotetext{
${ }^{* 1}$ This Paper was Originally Published in Japanese in J. Japan Inst. Met. Mater. 81 (2017) 66-70.

${ }^{* 2}$ Graduate Student, Kyushu Institute of Technology
}

sical MD method, which assumes an appropriate interatomic interaction, the so-called empirical potential. The former can be used to perform highly accurate calculations, but enormous calculation time is necessary. In contrast, the latter can deal with large-scale systems, but its accuracy is inferior to that of the first principles method. Regarding research on structural relaxation, long-period, large-scale simulation is required, so the classical MD method is most effective. In the present study, we prepared $a$-Ge by a MD approach using the Tersoff potential, and confirmed the validity of this method. Subsequently, the structural changes in $a$-Ge during thermal annealing were investigated.

\section{Simulation Methods}

An empirical interatomic potential proposed by Tersoff ${ }^{7)}$ was employed for MD simulations. Although this potential is known to produce a melting point higher than the experimental value, it is able to reproduce the static and dynamic behaviors of $\mathrm{Si}$ and $\mathrm{Ge}$ in the liquid and amorphous states ${ }^{8-13)}$. $1000 \mathrm{Ge}$ atoms were arranged into a diamond structure in the MD cell with a constant volume of periodic boundary condition $5 a_{0} \times 5 a_{0} \times 5 a_{0}\left(a_{0}\right.$ is the lattice constant of Ge: $0.5658 \mathrm{~nm}$ ). This cell was held at $3500 \mathrm{~K}$ for 100 ps to prepare liquid $\mathrm{Ge}$. The liquid was rapidly quenched to $300 \mathrm{~K}$ at a cooling rate of $10^{12} \mathrm{~K} / \mathrm{s}$ and held at $300 \mathrm{~K}$ for $10 \mathrm{ps}$ to produce $a$-Ge (as-quenched $a-\mathrm{Ge}$ ). This as-quenched $a$-Ge was held at temperatures ranging from $900 \mathrm{~K}$ to $1800 \mathrm{~K}$ for $10 \mathrm{~ns}$ and again quenched to $300 \mathrm{~K}$ at a rate of $10^{12} \mathrm{~K} / \mathrm{s}$ (annealed $a-\mathrm{Ge})$. For the simulation, the time step was set to $2 \mathrm{fs}$ and the temperature was controlled by the velocity scaling method.

\section{Results and Discussion}

It has been reported that the melting point of Ge produced by the Tersoff potential is $2448 \mathrm{~K}^{14)}$, and therefore it was confirmed that steady state liquid $\mathrm{Ge}$ is obtained by holding at $3500 \mathrm{~K}$ for $100 \mathrm{ps}$. Figure 1 shows the potential energy change during cooling at $10^{12} \mathrm{~K} / \mathrm{s}$. The fluctuation in the potential energy decreases with cooling, which corresponds to 
the reduction in thermal atomic vibration. The potential energy initially decreases monotonically with cooling, but drops more sharply at $2500 \mathrm{~K}$ and then decreases linearly below $\sim 1600 \mathrm{~K}$, suggesting that glass transition occurs at about $1600 \mathrm{~K}$. The existence of a glass transition point has been experimentally confirmed in amorphous $\mathrm{Si}$, which like $\mathrm{Ge}$ is a group IV semiconductor ${ }^{15}$ ). It is empirically known that the ratio of the glass transition point $T_{\mathrm{g}}$ to the melting point $T_{\mathrm{m}}$ $\left(T_{\mathrm{g}} / T_{\mathrm{m}}\right)$ is $2 / 3$. It has also been experimentally confirmed that the glass transition point of $\mathrm{Ge}$ is $750 \mathrm{~K}$ and its melting point is $1210 \mathrm{~K}^{16)}$, giving $T_{\mathrm{g}} / T_{\mathrm{m}}=0.62$, which is close to the empirical rule. From our simulations, $T_{\mathrm{g}} / T_{\mathrm{m}}$ is equal to 0.65 , in agreement with the experimental value. This suggests that the Tersoff potential can qualitatively reproduce the glass transition behavior, though its melting temperature $(2448 \mathrm{~K})$ differs from the experimental value.

To examine the effects of annealing temperature on the amorphous structure, as-quenched $a$-Ge was held at temperatures ranging from $900 \mathrm{~K}$ to $1800 \mathrm{~K}$. The change in the potential energy as a function of annealing time is shown in Fig. 2. The potential energy during annealing at $900 \mathrm{~K}$ does not change with respect to time, and no structural relaxation occurs. This is attributed to $900 \mathrm{~K}$ being insufficient to induce

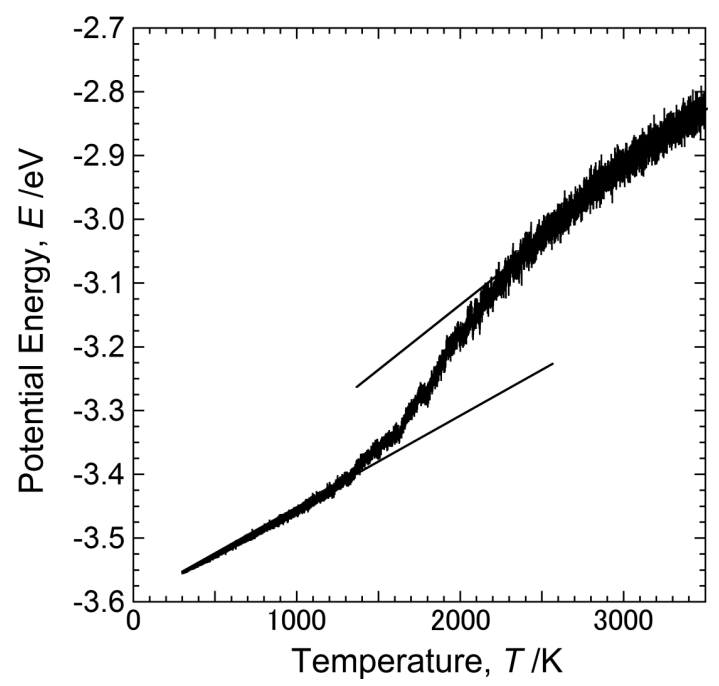

Fig. 1 Change in potential energy during quenching at cooling rate of $10^{12} \mathrm{~K} / \mathrm{s}$.

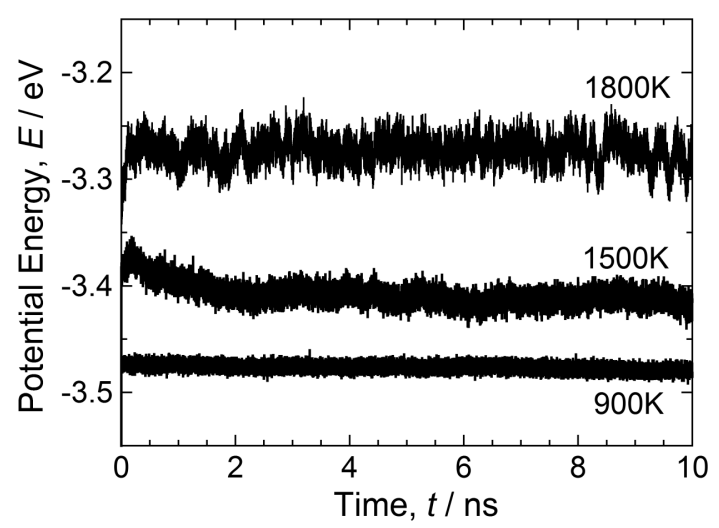

Fig. 2 Change in potential energy during thermal annealing at 900, 1500, and $1800 \mathrm{~K}$. atomic rearrangement and bond switching for structural relaxation. However, the potential energy decreases with increasing annealing time at $1500 \mathrm{~K}$. This suggests that defects and strains present in the as-quenched network disappear and structural relaxation occurs. At $1800 \mathrm{~K}$, the potential energy rises during the initial stage and is then constant. The glass transition point was estimated to be $\sim 1600 \mathrm{~K}$ (Fig. 1), and therefore the amorphous material is molten at $1800 \mathrm{~K}$.

We investigated the structure of $a$-Ge obtained after annealing at $1500 \mathrm{~K}$. Figure 3(a) shows the radial distribution functions obtained from the atomic arrangement of asquenched (broken line) and annealed $a$-Ge (solid line). The annealed atomic arrangement was obtained by maintaining as-quenched $a$-Ge at $1500 \mathrm{~K}$ for $10 \mathrm{~ns}$ and then quenching it to $300 \mathrm{~K}$ at a cooling rate of $10^{12} \mathrm{~K} / \mathrm{s}$. The first and second peaks in Fig. 3(a) exist at $0.248 \mathrm{~nm}$ and $0.406 \mathrm{~nm}$, respectively, whereas no significant peak is observed at $0.469 \mathrm{~nm}$, which is the third nearest interatomic distance of crystalline Ge. These features of the radial distribution function agree with those obtained experimentally ${ }^{17,18}$. The present MD simulations were performed under the constant volume condition, but it was confirmed that a similar structure can be obtained even in constant pressure simulations. The height of the first peak is hardly changed by annealing, while the second peak becomes large. Similar structural changes have been reported for amorphous $\mathrm{Si}$, the structure of which is similar to that of $\mathrm{Ge}^{19)}$, suggesting that our simulation reproduces realistic structural relaxation.

Figure 3(b) shows the bond angle distributions of asquenched (broken line) and annealed $a$-Ge (solid line). In
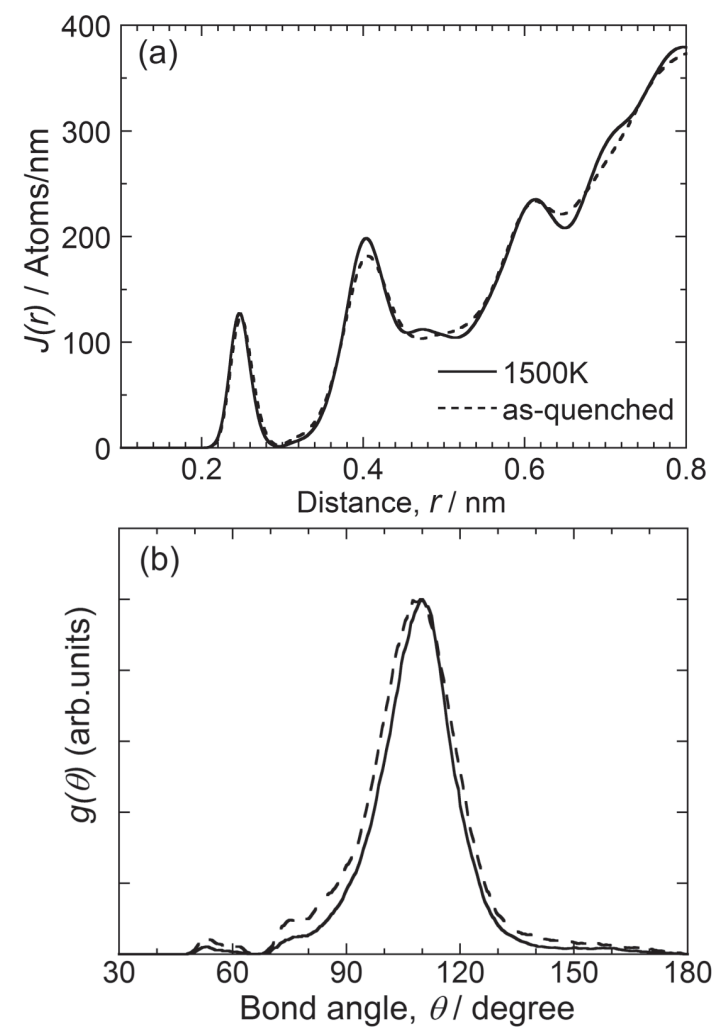

Fig. 3 (a) Radial distribution functions and (b) bond angle distribution functions of as-quenched (broken line) and $1500 \mathrm{~K}$-annealed amorphous Ge (solid line). 
both cases, a peak exists at $\sim 109.5^{\circ}$, indicating that the amorphous network consists of a tetrahedral structure as a shortrange order. Additionally, the peak width of bond angle distribution decreases with annealing. The standard deviation of the bonding angle, $\Delta \theta$, of as-quenched and annealed $a-\mathrm{Ge}$ is $12.5^{\circ}$ and $11.0^{\circ}$, respectively. Because strain energy is proportional to $\Delta \theta^{20)}$, the strain energy decreases with annealing, which is consistent with structural relaxation. The reduction of $\Delta \theta$ caused by structural relaxation has also been confirmed by Raman spectroscopy ${ }^{21)}$.

Figure 4(a) shows the change in potential energy during annealing at $1200 \mathrm{~K}$, sufficiently lower than the glass transition point. As observed for $1500 \mathrm{~K}$ annealing, the potential energy decreases and structural relaxation takes place. It should be noted that undulation exists between 4.3 and $5.6 \mathrm{~ns}$; the low potential state at $4.3 \mathrm{~ns}$ transforms to another one at $5.6 \mathrm{~ns}$ via the high potential state at $5.1 \mathrm{~ns}$. A similar change is seen at $1500 \mathrm{~K}$ (Fig. 2), but its frequency is larger. To clarify the origin of this potential energy undulation, the mean square displacement (MSD) was investigated. When a particle existing at $r_{\alpha}\left(t_{0}\right)$ at time $t_{0}$ moves to $r_{\alpha}\left(t+t_{0}\right)$ after the elapse of time $t$, the MSD can be calculated using the following equation:

$$
\left\langle R^{2}(t)\right\rangle=\frac{1}{N} \sum_{\alpha=1}^{N}\left[r_{\alpha}\left(t+t_{0}\right)-r_{\alpha}\left(t_{0}\right)\right]^{2},
$$

where $N$ is the number of particles. Figure 4(b) shows the time evolution of the MSD at $1200 \mathrm{~K}$. The existence of plateau regions in the MSD reveals that no melting occurs during annealing ${ }^{22)}$. In contrast, the MSD greatly changes during $4.3 \mathrm{~ns}$ and $5.6 \mathrm{~ns}$ where the undulation of the potential energy
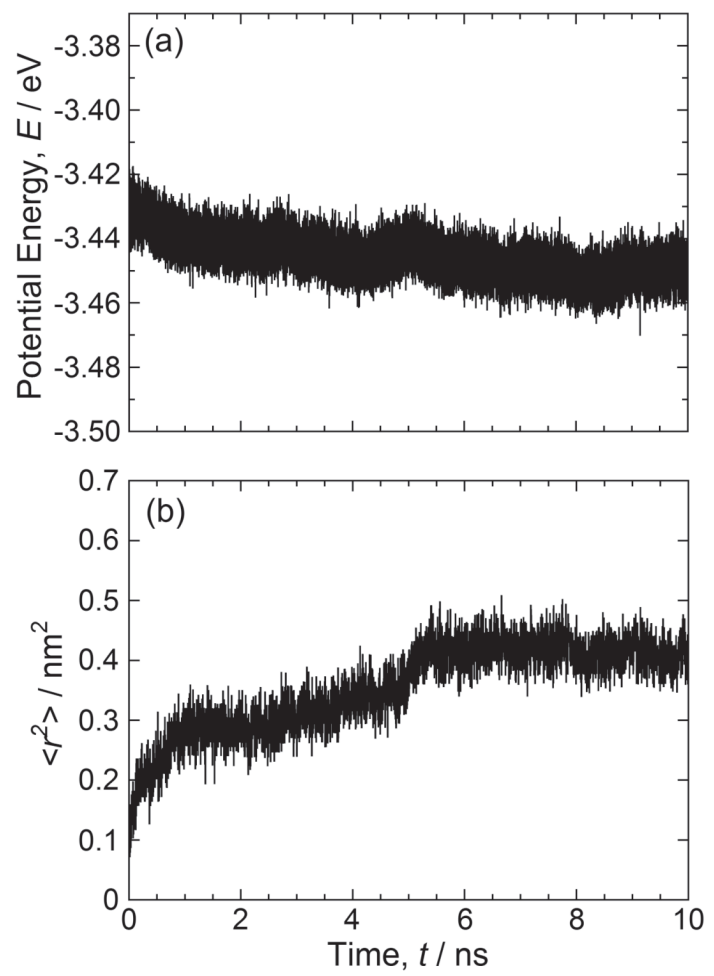

Fig. 4 (a) Potential energy and (b) mean square displacement (MSD) as a function of annealing time. The annealing temperature of the system is $1200 \mathrm{~K}$. was observed in Fig. 4(a), indicating the occurrence of remarkable atomic movement.

To obtain information on the atomic motion, the atomic positions during annealing at $1200 \mathrm{~K}$ are shown in Fig. 5. We plotted atoms contained in the range of $5 a_{0}$ in the $x$ and $y$ directions and $a_{0}$ in the $z$ direction over 0 to $10 \mathrm{~ns}$. In addition to the atoms thermally vibrating on the lattice points, moving atoms largely exist in the region of $x=1.2$ to $2.0 \mathrm{~nm}$ and $y=$ 1.0 to $2.5 \mathrm{~nm}$. Because the movement of atoms during the whole simulation time ( 0 to $10 \mathrm{~ns}$ ) is plotted in Fig. 5, the movement of each atom is difficult to see. Therefore, we next investigated the movement of atoms in a shorter period. Figure 6(b) shows the trajectory of atoms in the time range from 4.3 to $5.6 \mathrm{~ns}$, in which large changes were observed in the potential energy (Fig. 4 (a)) and MSD (Fig. 4 (b)). For comparison, the trajectories of atoms at 1.0 to $2.3 \mathrm{~ns}$ and 8.0 to 9.3 ns are shown in Fig. 6(a) and (c), respectively. In Fig. 6(b), $\mathrm{F}$ is the trajectory of only the atoms moving less than $0.25 \mathrm{~nm}$, which is approximately the first nearest neighbor distance of $\mathrm{Ge}$, while A to $\mathrm{E}$ are trajectories of atoms moving more than $0.25 \mathrm{~nm}$. In Fig. 6(a) and (c), it can be seen that the majority of the atoms thermally vibrate on the lattice points. In contrast, the atomic positions of $\mathrm{A}$ to $\mathrm{E}$ are quite different between Fig. 6(a) and (c). It is clear in Fig. 6(b) that A to E atoms migrate significantly between 4.3 and $5.6 \mathrm{~ns}$ where remarkable changes were observed in the potential energy and MSD (Fig. 4).

To investigate the correlation between the motions of different atoms, Fig. 7 shows the square displacement of A to E atoms, which showed large-scale movements in Fig. 6(b). For comparison, the result for the $\mathrm{F}$ atom vibrating on the lattice points is also shown. The position of the origin of the vertical axis is shifted in Fig. 7. The open and filled triangles indicate the start and end times of the large-scale movements, respectively. The atomic movements become more pronounced locally during the time period in which remarkable changes are observed in the potential energy and MSD (Fig. 4). In other words, structural changes are induced by spatially- and temporally-inhomogeneous atomic motions; atomically mobile and immobile regions coexist during structural relaxation. It should be noted that the fluctuation in the square displace-

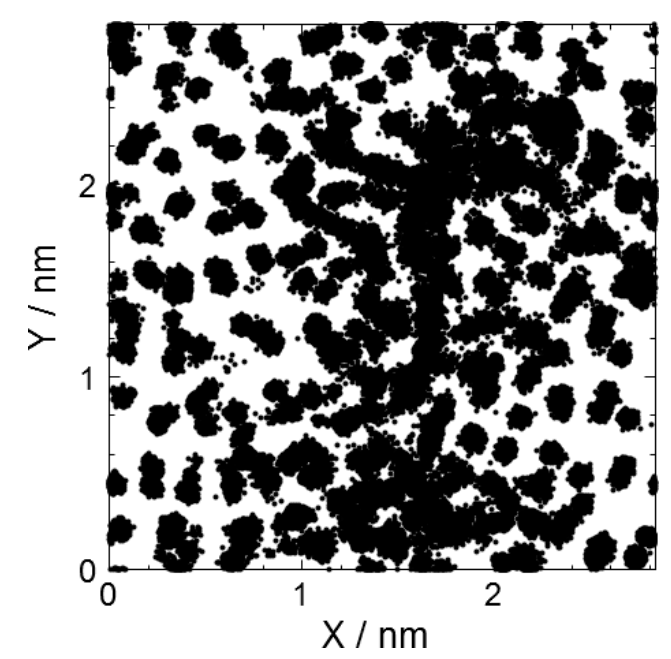

Fig. 5 Atomic trajectories in a-Ge annealed at $1200 \mathrm{~K}$ for $10 \mathrm{~ns}$. 
(a)
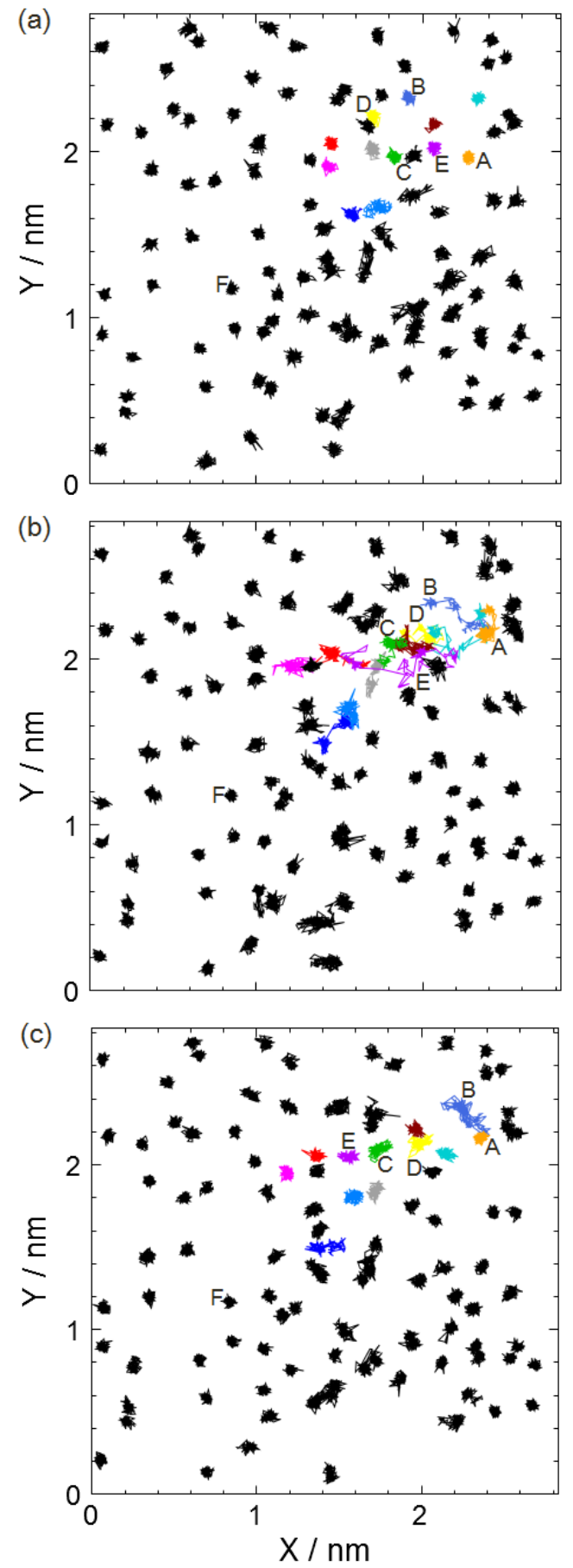

Fig. 6 Atomic trajectories in a-Ge annealed at $1200 \mathrm{~K}$ for (a) $1.0-2.3 \mathrm{~ns}$, (b) 4.3-5.6 ns, and (c) 8.0-9.3 ns.

ment of $\mathrm{A}$ to $\mathrm{E}$ atoms is larger than that of the $\mathrm{F}$ atom vibrating at the lattice points. The reason for this is not clear, but it is suggested that $\mathrm{A}$ to $\mathrm{E}$ atoms are intrinsically easy to move, even before and after the large-scale atomic displacement occurs. Similar cooperative atomic motions have also been observed in the structural relaxation of metallic glasses ${ }^{23)}$. In a supercooled liquid state, a region where atoms are easy to move and a region where atoms are difficult to move are reported to coexist ${ }^{24-26)}$. This phenomenon is known as dynamic heterogeneity. In the present simulation, we investigated the behavior of $a$-Ge below the glass transition temperature, but behavior similar to dynamical heterogeneity was observed

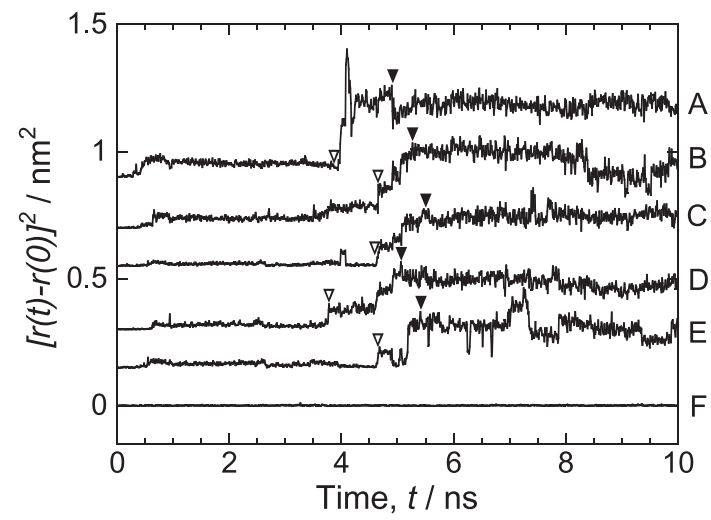

Fig. 7 Square displacements of atoms A-F in Fig. 6. A-E: atoms existing in remarkable atomic mobile region; F: atoms thermally vibrated on the lattice site.

during the structural relaxation.

\section{Conclusions}

The structure of $a$-Ge and its behavior during thermal annealing were examined by MD simulations based on the empirical Tersoff interatomic potential. The following results were obtained:

(1) A liquid-to-glass transition occurred at $\sim 1600 \mathrm{~K}$ during quenching. Its value is $\sim 2 / 3$ of the melting temperature, which agrees with the experimental result. It was confirmed that the calculated radial distribution function and bond angle distribution of $a-\mathrm{Ge}$ and their change caused by structural relaxation well reproduce the experimental results. This suggests that the Tersoff potential is useful for investigating the structure and structural relaxation processes of $a-\mathrm{Ge}$, even though the melting and glass transition temperatures it produces are higher than the experimental values.

(2) The as-quenched $a$-Ge was annealed at temperatures ranging from $900 \mathrm{~K}$ to $1800 \mathrm{~K}$. It was confirmed that the amorphous state is molten at $1800 \mathrm{~K}$, because its temperature is higher than the glass transition temperature $(\sim 1600 \mathrm{~K})$. The potential energy decreases with thermal annealing at $1200 \mathrm{~K}$ and $1500 \mathrm{~K}$, suggesting that defects and distortions present in the as-quenched a-Ge network disappear and structural relaxation takes place. The migration of atoms is insufficient at $900 \mathrm{~K}$, and so no remarkable structural relaxation is observed.

(3) From the trajectory of atoms and the mean square displacement of atoms, it was found that spatially- and temporally-inhomogeneous structural changes occur during the structural relaxation of $a$-Ge. That is, in addition to the atoms vibrating on lattice points, there is a region in which atoms migrate collectively on a large scale. This behavior is similar to the dynamic heterogeneity observed in supercooled liquids.

\section{REFERENCES}

1) H. Wu and Y. Cui: Nano Today 7 (2012) 414-429.

2) M. Tane, S. Nakano, R. Nakamura, H. Ogi, M. Ishimaru, H. Kimizuka and H. Nakajima: Acta Mater. 59 (2011) 4631-4640.

3) R. Nakamura, M. Ishimaru, A. Hirata, K. Sato, M. Tane, H. Kimizuka, T. Shudo, T.J. Konno and H. Nakajima: J. Appl. Phys. 110 (2011) 064324. 
4) R. Nakamura, M. Ishimaru, K. Sato, K. Tanaka, H. Nakajima and T.J. Konno: J. Appl. Phys. 114 (2013) 124308.

5) M. Okugawa, R. Nakamura, M. Ishimaru, K. Watanabe, H. Yasuda and H. Numakura: J. Appl. Phys. 119 (2016) 214309.

6) M. Okugawa, R. Nakamura, M. Ishimaru, H. Yasuda and H. Numakura: AIP Adv. 6 (2016) 125035.

7) J. Tersoff: Phys. Rev. B 39 (1989) 5566-5568.

8) M. Ishimaru, K. Yoshida and T. Motooka: Phys. Rev. B 53 (1996) 7176-7181.

9) M. Ishimaru, K. Yoshida, T. Kumamoto and T. Motooka: Phys. Rev. B 54 (1996) 4638-4641.

10) M. Ishimaru, S. Munetoh and T. Motooka: Phys. Rev. B 56 (1997) 15133-15138.

11) M. Ishimaru: J. Appl. Phys. 91 (2002) 686-689.

12) M. Ishimaru, M. Yamaguchi and Y. Hirotsu: Phys. Rev. B 68 (2003) 235207.

13) J.K. Bording: Phys. Rev. B 62 (2000) 7103-7109.

14) P. López, L. Pelaz, I. Santos, L.A. Marqués and M. Aboy: J. Appl. Phys. 111 (2012) 033519.

15) A. Hedler, S.L. Klaumunzer and W. Wesch: Nat. Mater. 3 (2004) 804
809.

16) T. Masumoto, K. Suzuki, H. Huzimori and K. Hashimoto: Amorphous kinnzoku no kiso, (Ohmsha, Tokyo, 1982) p. 36.

17) G. Dalba, P. Fornasini, M. Grazioli and F. Rooca: Phys. Rev. B 52 (1995) 11034-11043.

18) S. Roorda, C. Martin, M. Droui, M. Chicoine, A. Kazimirov and S. Kyucia: Phys. Rev. Lett. 108 (2012) 255501.

19) K. Laaziri, S. Kycia, S. Roorda, M. Chicoine, J.L. Robertson, J. Wang and S.C. Moss: Phys. Rev. Lett. 82 (1999) 3460-3463.

20) P. Roura, J. Farjas and P.R.I. Cabarrocas: J. Appl. Phys. 104 (2008) 073521.

21) F. Kail, J. Farjas, P. Roura, C. Secouard, O. Nos, J. Bertomeu, F. Alzina and P.R.I. Cabarrocas: Appl. Phys. Lett. 97 (2010) 031918.

22) M. Ishimaru: Phys. Rev. B 63 (2001) 237401.

23) M. Shimono and H. Onodera: Mater. Sci. Forum 638-642 (2010) 1648-1652.

24) M.D. Ediger: Annu. Rev. Phys. Chem. 51 (2000) 99-128.

$25)$ W. Kob, C. Donati, S.J. Plimpton, P.H. Poole and S.C. Glotzer: Phys. Rev. Lett. 79 (1997) 2827-2830.

26) R. Richert: J. Phys. Condens. Matter 14 (2002) R703-738. 